\title{
Steven Vande Moortele, The Romantic Overture and Musical Form from Rossini to Wagner, Cambridge: Cambridge University Press 2017
}

Schlagworte/Keywords: 19. Jahrhundert; 19th century; Gattungsgeschichte; history of musical genre; musical form; musikalische Form; Ouvertüre; overture

Eine wissenschaftliche Annäherung an die Form der romantischen Ouvertüre vorzulegen, erfordert einen metatheoretischen Ansatz oder, mit den Worten des Autors selbst, das Bewusstsein einer prima und einer seconda pratica der Formenlehre (11f.; 220f.) in der ersten Hälfte des 19. Jahrhunderts. Mit diesem Rückgriff auf ein theoretisches Konstrukt aus dem frühen Barock ist die spannungsvolle Koexistenz zwischen den Standards der klassischen Sinfonie und ihren romantischen Überformungen gemeint. Da letztere nicht linear im Sinne eines Fortschreitens stilistischer Modernität stattfinden, sondern willkürlich erfolgen können, ist ein solcher Rückgriff auf ein Beschreibungsmodell für ein Nebeneinander mehr oder weniger normativer Standards sehr sinnvoll. In aktueller Theorie sind die Standards klassischer Form in der ,New Formenlehres durch William Caplin, ${ }^{1}$ James Hepokoski und Warren Darcy ${ }^{2}$ sowie in Janet Schmalfeldts Buch In the Process of Becoming ${ }^{3}$ wissenschaftlich erfasst (1f.). Somit stellt Steven Vande Moorteles Studie nicht allein eine analytische Auseinandersetzung mit fast 200 Ouvertüren dar, sondern auch eine Anwendung und »Rekalibrierung « (10) der >New Formenlehre` auf ein zwar verwandtes, aber dennoch eigene formale Räume ausprägendes Repertoire. Dabei übt Vande Moortele eingangs Kritik an dem einer etwas zu reinen Strukturanalyse verpflichteten Ansatz in der Sonatentheorie Hepokoskis und Darcys, in dem Probleme der musikalischen Gattung keine Rolle spielen (2). Wie Adolph Bernhard Marx nach Carl Dahlhaus eigentlich eine Gattungslehre der Klaviersonate erstellt hat und keine Sonatentheorie, so hätte sich demnach diese Indifferenz gegenüber den formalen Merkmalen einer Gattung bei Hepokoski und Darcy erhalten (2).

\footnotetext{
1 Caplin 1998.

2 Hepokoski/Darcy 2006.

3 Schmalfeldt 2011.
}

\section{Die OUVERTÜre Als GATTUNG IM}

\section{JAHRHUNDERT}

So handelt das erste Kapitel »Making Overtures« (15-45) vom Aufstieg der Gattung Ouvertüre in den ersten Jahrzehnten des 19. Jahrhunderts, von ihrem spannungsvollen Verhältnis zur Sinfonie und von ihrer Emanzipation gegenüber einer Sinfonik, die keine eröffnende Funktion für ein folgendes Stück - Theater oder Oper - mehr hat, sondern zu jedem Zeitpunkt eines Konzertabends gespielt werden kann. Untersuchungszeitraum und -ort bilden dabei diejenigen geschichtlichen Daten, die fachkundigen Personen in den Musikstädten Leipzig und Berlin zwischen 1815 und 1830 bekannt sein konnten (6). Die Auswahl gerade dieser norddeutschen Perspektive begründet Vande Moortele mit der europaweiten Vernetzung der beiden Städte und mit der Prominenz von Ouvertüren in Konzert- und Theaterprogrammen sowie im musikwissenschaftlichen Diskurs der einschlägigen Zeitschriften, vor allem der Leipziger und Berliner Allgemeinen Musikalischen Zeitung, der Neuen Zeitschrift für Musik und der Zeitschrift Iris im Gebiete der Tonkunst. Das kosmopolitische Musikleben in diesen beiden Städten erlaube es, über eine gewisse germanozentrische Orientierung hinaus (8) die Gattung Ouvertüre an ihrem adäquaten musikgeschichtlichen Ort zu erfassen, nämlich als europäische Instrumentalmusik, in der Probleme sinfonischen Komponierens und sinfonischer Form innovativ und nicht selten auf experimentellem Wege weiterentwickelt wurden.

Straddled between the theater and the concert hall, the overture can even be considered the only truly European instrumental genre of its time. From a Germanic perspective it bridges the gap between Beethoven's symphonies and Liszt's symphonic poems in 
an era plagued by recurring doubts about the viability of the symphony. From a broader European point of view, its close association with opera allowed the overture to flourish even in those countries where independent traditions of instrumental music where otherwise marginal, such as France and Italy. (6)

Die Form der Ouvertüre als eine spezifisch romantische wird auf dieser Grundlage weniger als Erosion oder ssetzender، Gegenentwurf zu klassischen Formmodellen dargestellt, sondern als ein Feld möglicher Konstellationen aus traditionellen Vorlagen, intertextuellen Bezügen, Ansprüchen der Gattung, sich als Standard etablierenden Innovationen und der zeitgenössischen Theorie.

Als Schlüsselereignis nennt Vande Moortele die Aufführung aller drei Leonoren-Ouvertüren und der Ouvertüre zu Fidelio durch das Gewandhausorchester Leipzig unter der Leitung von Felix Mendelssohn Bartholdy am 9. Januar 1840. Dieses im Untersuchungszeitraum eher spät liegende Konzert spiegele den neuen Status der Ouvertüre als Konzertstück im Range einer Sinfonie wieder (15-17).

\section{RELATIVIERUNG ORGANIZISTISCHER FORMTHEORIEN}

Nicht allein in den Reaktionen auf dieses Konzertereignis in der zeitgenössischen Kritik (16), sondern vor allem im Vergleich zwischen Ouvertüre und Sinfonie relativiert Vande Moortele die weitgehend auf Organismusmetaphern beruhende Vorstellung klassischer und romantischer Form, die Nachahmung einer natura naturans ${ }^{4}$ als alleinigen Maßstab ästhetischer Vollkommenheit. Damit rekurriert er wiederum auf Marx als Schlüsselfigur romantischer Formenlehre, aber auch auf die Aufarbeitung und teilweise Verfestigung organizistischer Formtheorien in der jüngeren Musiktheorie und Musikwissenschaft. ${ }^{5}$ Eine adäquate Auseinandersetzung mit sogenannten Potpourri-Ouvertüren oder eher schablonenhaft komponierten Ouvertüren im Stile Rossinis werde durch abwertende, auf dem Organismusmodell beruhende Vorurteile erschwert (50).

5 Bonds 1991; Kawohl 2005; Rigaudière 2009.
While the organic artwork is well made, the mechanical is not. "Well made," in this context, refers to the poetic as well as the aesthetic aspect: mechanical (and, therefore, nonorganic) is that which is put together in such a manner that the final product still looks as if it has been put together. Its parts are insufficiently integrated, it lacks individuality, and it fails to express an underlying idea. (50)

Eine Relativierung dieser pejorativen Haltung gegenüber eher reihenden, mechanisch anmutenden Formen sei notwendig, um bestimmten Ausprägungen romantischer Form überhaupt in der Analyse gerecht zu werden (51). Vande Moorteles Analysen von Rossinis Formbehandlung nach dem Modell der Ouvertüre zu La Cenerentola sind demzufolge nicht zwingend auf einer Vorstellung von Form als Prozess aufgebaut, in der spätere Motive als Transformationen und Ableitung früher exponierter Motive den Eindruck von Wachstum und organischer Einheit suggerieren, was im Falle Rossinis, der bekanntlich und nach eigener Aussage Motive, Themen und ganze Ouvertüren mehrfach verwandte, auch kaum sinnvoll wäre (56). Die in dieser Hinsicht neutralere Nomenklatur der 'New Formenlehre erlaubt demnach einen Zugang zu Rossini (51), der aufbauend auf den älteren Analysen Philip Gossetts $^{6}$ - von einer um bestimmte Züge erweiterten Sonatinen-Form ausgeht und dabei als entscheidendes Merkmal das sogenannte 'Rossini-Crescendor herausarbeitet. Dieser affirmative, meist eingängige und verhältnismäßig ausgedehnte Formteil im orchestralen Tutti im Anschluss an die Seitensatzgruppe (subordinate theme) ist nach Vande Moortele das subversivste Element in Rossinis Formbehandlung (56) und erscheint im Buch als erste einer ganzen Reihe sstarker Erweiterungen klassischer Modelle.

Auch die Betrachtung der Potpourri-Ouvertüren profitiert vom Ausblenden einer Wertung a priori durch Organismus-Metaphern. Da auch Maßstäbe setzende Kompositionen wie die Ouvertüre zum Freischütz (1821) und zum Oberon (1826) von Carl Maria von Weber wie auch die Ouvertüre zum Sommernachtstraum (1826) von Felix Mendelssohn Bartholdy der zeitgenössischen Kritik einer zu parataktischen, 
die Einheit des Werks nur wenig beachtenden, Verkettung durch von außen hinzugefügte Narrative in verschiedenem Ausmaß ausgesetzt waren, lässt sich Vande Moorteles Kapitel zu den Potpourri-Ouvertüren (75-107) als Rehabilitation dieses Formtyps verstehen. Auch hier geht es zwar u. a. um eine ästhetische Würdigung, im Zentrum scheint jedoch eine Verschiebung analytischer Paradigmen zu stehen: Eher einem prozessualen Werkverständnis verpflichtete Analysekriterien wie motivische Ableitung, Transformation und entwickelnde Variation werden durch Modelle ersetzt, die entsprechend einer gewissen Grundhaltung der formal functions der 'New Formenlehres - auf weniger teleologische Weise als die ältere Formenlehre auf planvoller Wiederholung und Wiederholbarkeit, Rekomposition sowie eingelöster oder nicht eingelöster Symmetrie beruhen.

Besonders zum Tragen kommt dieser Paradigmenwechsel bei den Analysen der motivischen Substanzgemeinschaft der meisten Themen in der Ouvertüre zum Sommernachtstraum (86-92) durch ein gemeinsames, meistens trunkiertes Tetrachord e-dis-c*-h. In den analytischen Beiträgen zur 'Submotivikı durch Dahlhaus und Friedhelm Krummacher ${ }^{7}$ wäre eine solche Substanzgemeinschaft dialektisch zu verstehen, als Indiz für eine subkutane, prozessuale Logik, in der äußerlich disparate formale Elemente dennoch einer durch die dialektische Anstrengung gestärkten Idee organischer Einheit verpflichtet sind. Im dialektischen Nachvollzug zwischen äußerlicher Disparatheit und innerem Zusammenhang durch submotivische Bezüge stellen sich nach diesem Ansatz relevante Wirkungen formaler Ästhetik besonders in Beethovens Spätwerk ein, aber eben auch mit gewissen Modifikationen in Mendelssohns Formensprache. Vande Moortele konfrontiert dieses induktive Verständnis von Form über motivische Bezüge mit einem eher räumlichen Verständnis von Form als Organisation zeitlicher Funktionen. Die Substanzgemeinschaft durch das Tetrachord gebe per se keinerlei Information über die Reihenfolge der motivisch-thematischen Varianten, und insgesamt sei die Existenz dieser motivischen Basis auch nicht unbedingt eine Garantie für eine Auskomposition im Sinne organischer Einheit.

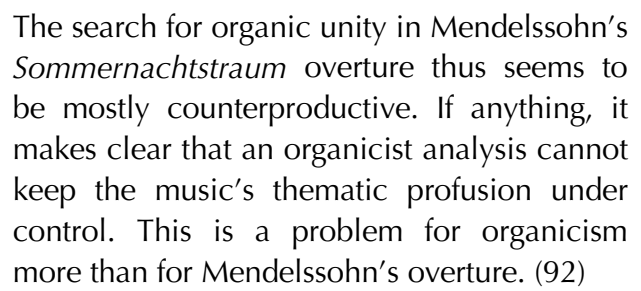

Obwohl diese Abfertigung eines recht großen Feldes deutschsprachiger Analysekultur hier auf sehr schnellem Wege erfolgt, bleibt doch ein analytischer Gewinn zurück, nämlich in der Idee, dass es alternative Vorstellungen und Begründungen für die Existenz submotivischer Bezüge in Mendelssohns Komponieren gibt, zumal da ein Mangel an organizistischen Formeigenschaften wie eine zu schwache Ausprägung von Wachstumsmetaphern zum Tenor der nicht nur zeitgenössischen Kritik an Mendelssohn gehört. ${ }^{8}$

Ebenso scheint es einem Zurückhalten organizistischer Wertungen zu verdanken zu sein, dass Vande Moortele ein formales Element, das er als »localized sonaticization« (98) beschreibt, in einer vergleichenden Analyse der Ouvertüre zur Oper Zampa von Ferdinand Hérold, der Ouvertüre zu Guillaume Tell von Rossini und der in der öffentlichen Wahrnehmung ungleich sublimeren Ouvertüre zum Fliegenden Holländer von Richard Wagner erarbeitet. Dabei wird 'Sonatenform' eben nicht als übergeordnetes Prinzip, sondern als punktueller formaler Marker in einer sonst reihenden Logik verstanden:

As we saw at the beginning of this chapter, potpourri form is usually defined only in negative terms, that is, as the absence of form in the emphatic sense. [...] A more positive approach is to think of the juxtaposition of contrasting tableaux itself as a formal principle. (98)

"Localized sonaticization « sind demnach formale Maßnahmen wie Modulationen, quasirückführende Orgelpunkte, Kadenzbildungen, typische Instrumentationen oder andere wiedererkennbare Elemente sonatenhaften Komponierens, die sich verfügbar machen lassen und in andere Formabläufe integriert werden. Nicht allein aufgrund derartiger Elemente weist 
Vande Moortele nach, dass nicht einmal in der Ouvertüre zu Zampa, diesem seinerzeit ungemein beliebten und dabei für seine Form vielfach verrissenen Stück (92), zu Recht von einer bloßen Reihung von Themen gesprochen werden kann (93-100).

\section{STARKE FORMTEILE}

Die vier folgenden Kapitel »Beginning before the Beginning (105-145), »Strong Subordinate Themes« (146-190), »Open-Ended Expositions « (191-221) und »Recomposed Recapitulations« (222-259) beschreiben zum einen das Aufweichen kritischer Formgrenzen der Sonatenform, d. h. zwischen langsamer Einleitung und Exposition, zwischen Exposition und Durchführung sowie zwischen Durchführung und Reprise, zum anderen das Aufblühen neuer, meist sstarker`Formteile, für die das >Rossini-Crescendo< eine Art Vorbote war. Im Einzelnen sind dies "starke Seitenthemen" (strong subordinate themes; 146), „Durchbruch«-Modelle in Abwandlung von Theodor W. Adornos Begriffsprägung (224f.) und "Apotheosen« (apotheoses; 240-247). Vande Moortele erfasst diese formalen Innovationen in einem übergeordneten, an Kofi $\mathrm{Agawu}^{9}$ und William Caplin ${ }^{10}$ angelehnten »heuristischen Modell« (112) von Zeitfunktionen. Der äußerliche Grund für die Einführung einer übergeordneten systematischen Betrachtung formaler Strategien ist die in zwei Richtungen ausstrahlende Wirkung langsamer Einleitungen auf die folgende Ouvertüre: Je länger die vorangehende Einleitung ist, desto eher wirkt sie snach außen vertüre, aber auch ınach innenı, da sie selbst zum eigenständigen Musikstück wird. Angesichts dieser Abhängigkeit formaler Bestimmung von grundlegenden Faktoren zeitlicher Natur, ist eine Betrachtungsebene notwendig, die den Maximen klassischer oder romantischer Formenlehre übergeordnet ist:

I propose as a tool for analyzing these introductions the heuristic model of a complete and rounded musical form. This heuristic model, which consolidates some of Caplin's and Hepokoski and Darcy's observations

10 Vgl. Caplin 1998, 15, V. about classical slow introductions, comprises five functionally and temporally distinct units: prefatory (before the beginning); initiating (beginning); medial (middle); closing (end); and epilogic (after-the-end). These units are articulated and differentiated by means of phrase-structural, cadential, and topical (i.e., melodic-motivic) organization. (112)

Varianten einer solchen zeitlich-funktionalen Betrachtungsebene werden auch in den folgenden Kapiteln, die über die Introduktion hinausgehen, immer wieder angewandt und demonstrieren so etwas wie »privilegierte Zonen ${ }^{11}$ für formale Erweiterungen oder subversive Innovationen. Dabei sind einige neue Metaphern wie z. B. "Schwellen« (thresholds; 108111) besonders sprechend und dem musikalischen Inhalt angemessen, da sie die eigentliche, über die Tradition der klassischen Formenlehre weit hinausgehende Grunderfahrung des Beginnens in den analytischen Horizont miteinbeziehen.

Versucht man, nach der Lektüre des Buchs aus der gesammelten Materialfülle und den zahlreichen, sehr genauen Analysen auf implizite Grundaussagen rückzuschließen, so geben diese sstarken reiche Orientierung, da sie einen Zug der formalen Emphase ınach hinten`, an das Ende der Form plastisch nachvollziehbar machen. Vande Moortele lässt einen solchen finalen Zug, ein solches Verschieben in die hinteren Formteile, relativ häufig durchscheinen (166, 172, 180, 181, 189, 232, 240, 247ff.), ohne ihm ein eigenes Kapitel oder ein Fazit zu widmen; man kann jedoch davon sprechen, dass das Buch im impliziten >Abschreiten dells` prefatory-initiating-medial-closing-epilogic (112f.) diese Bewegung selbst vollzieht.

Innerhalb dieses Verschiebens formaler Emphase nach hinten ist den strong subordinate themes das umfangreichste Kapitel des Buches gewidmet (146-190). An Analysen besonders der Hebriden-Ouvertüre von Felix Mendelssohn Bartholdy, der Ouvertüre zum Fliegenden Holländer und der Ouvertüre zu Les Francs-Juges von Hector Berlioz werden Kriterien für diese `Stärke` von Seitensätzen erarbeitet und exemplifiziert, die sowohl aus sintrinsischer Perspektive - Lautstärke, Ausdehnung, 
thematische Kraft oder »thematicity « (166), Fasslichkeit oder »memorability« (164) - als auch aus sextrinsischer Perspektive bewertet werden. So findet Vande Moortele ausgesprochen überzeugende Worte dafür, dass zur 'Stärke` des subordinate theme aus Berlioz' Le Carnaval Romain auch gerade seine formale Position als subordinate theme gehört, die sich triumphal umkehrt, wenn mit diesem und nicht mit dem Hauptsatz die Reprise eingeleitet wird:

When, immediately after the development, it is the subordinate theme, not the beginning of the exposition, that launches the recapitulation, the masks come off. The strong subordinate theme now finally assumes the role it was supposed to play all along: that of a main theme. (180)

Gerade eine adäquate Bestimmung formaler Stärke ist nur in einer interaktiven Vorstellung von Formanalyse möglich, die Aspekte wie Hörerwartung, musikalisch durchlebte Zeit und das hörende Gedächtnis als dem Notentext gleichrangige Parameter behandelt.

[...] the relevance of any specific example of a strong subordinate theme remains limited when adduced without a close consideration of its immediate formal context. [...] The strength of a subordinate theme relies not only on what it is intrinsically, but also, and especially, on its relationship to the main theme. (190)

Als bemerkenswert ist das Kriterium »nach innen« (turning inward; 149) und »nach außen« (turning outward; 161) gerichteter Seitenthemen zu erwähnen. Vande Moortele eröffnet das Kapitel mit einem Exkurs zur Beschreibung von Seitenthemen bei Marx (146-148), von dem die setzende Zuordnung zu zeitgenössischen Gender-Modellen durch die Charakterisierung »männlich« für den Hauptsatz und "weiblich « ${ }^{12}$ für den Seitensatz stammt. Die berühmte Stelle bei Marx wird (auch) in dieser Publikation nicht vollständig zitiert - obwohl die Fortsetzung der Argumentation Vande Moorteles hier unterstützend entgegenkäme, denn auch Marx spricht in erster Linie von der gegenseitigen formalen Abhängigkeit beider Prinzipien:
Eben in solchem Sinn ist jeder der beiden Sätze ein Andres und erst beide miteinander ein Höheres, Vollkommneres.

Aber in diesem Sinn und der Tendenz der Sonatenform ist auch viertens begründet, dass beide gleiche Berechtigung haben, der Seitensatz nicht blos ein Nebenwerk, ein $\mathrm{Ne}$ bensatz zum Hauptsatz ist, mithin im Allgemeinen auch gleiche Ausbildung und gleichen Raum, wie der Hauptsatz fodert [...]. ${ }^{13}$

Im Sinne einer Idealisierung des Weiblichen in philosophischen Geschlechtertheorien des 19. Jahrhunderts, in denen wie z. B. an prominenter Stelle bei Wilhelm von Humboldt sowohl das rezeptive Vermögen als zwar introvertierter, aber durch die Fähigkeit fremde Vollkommenheiten aufzunehmen dem männlichen Prinzip überlegener Faktor hervorgehoben wird, ${ }^{14}$ wären zumindest die snach innen ausgerichteten sstarken seitenthemen in der Hebriden-Ouvertüre und in der Ouvertüre zum Fliegenden Holländer schlagende Beispiele für die bei Humboldt beschriebene kulturgeschichtlich relevante und im realpolitischen Sinne vor allem kontrafaktische Idee. In den Analysen wendet Vande Moortele durchaus verschiedene Argumentationen an, um die Wendung snach innen als musikalisch artikulierte nachzuweisen. Ist es in der Hebriden-Ouvertüre der durch die Stimmführung markierte Weg von der Dominante der Grundtonart h-Moll zur Seitensatztonart D-Dur, wobei der Halbtonschritt von fis nach $g$ (als Dominantseptime von A-Dur) eine sensible Rolle ${ }^{15}$ spielt (154f.), so ist es im Holländer die in der dramatischen Zuordnung des Hauptsatzes zum Außenbild des auf dem Meer treibenden Schiffes und des Sei-

13 Ebd.

14 Humboldt 1795, 119-122. Vgl. dazu Heinz 2002, 276-282. Zum Verhältnis von Marx' Sonatentheorie und Wilhelm von Humboldt vgl. Dahlhaus 1988, 347-364.

15 Dafür gibt es Vorbilder mit vergleichbarer inhaltlicher Konnotation, so in J. S. Bachs JOhannespassion im Choral Nr. 14 zum Text "Petrus, der nicht denkt zurück« oder auch in den Schlusstakten des Andantinos in fis-Moll der Sonate A-Dur D 959 (T. 189ff.) von Franz Schubert, wenn das leitereigene gis zu $g$ alteriert wird und damit eine sverinnerlichendes und innerhalb dieses Satzes singuläre Wendung nach D-Dur stattfindet. 
tensatzes zum Innenraum des Hauses erfolgende Bewegung aus der Landschaft in das häusliche Ambiente, mit der sich die Wendung snach innen vollzieht (156f.).

\section{FRAKTALE FORMSTRUKTUREN BEI WAGNER UND MENDELSSOHN}

Vande Moortele kommt bei der Analyse des Verhältnisses von langsamer Introduktion und Ouvertüre zu Rienzi zu Ergebnissen, die überzeugend auf formale Makrostrukturen verweisen, die eine zunächst als Großform verstandene formale Einheit auf einer nächsthöheren, die auditive Fasslichkeit zunächst übersteigenden formalen Hyper-Ebene verankern. Im Falle des Rienzi sind es die ersten 19 Takte der langsamen Einleitung, die zusammen mit den letzten vier Takten eine Klammer über die gesamte Ouvertüre bilden (125-127).

Wesentlich komplexer ist die fraktale Struktur, die Vande Moortele in der Analyse des Mottos des Sommernachtstraums herausarbeitet; nämlich dass durch eine solche motivische Präfiguration der später erfolgenden Themen der Eindruck entsteht, als seien Introduktion und Ouvertüre ineinander verwoben:

Thematic prefiguration and infiltration may seem rather crude ways of forging connections between a slow introduction and the rest of the overture [...]. Nonetheless, they are among the most striking techniques that can turn the introduction to an overture into a virtual overture en miniature. (137)

Die differenzierteste Darstellung eines solchen perspektivischen Formverständnisses findet sich dann schließlich in der Analyse der Ouvertüre zu Tannhäuser, in der Vande Moortele die Einbettung einer kleiner dimensionierten Sonatenform in eine größere nachweist, die beide in der Reprise zu einer Großform zusammenlaufen und so der Reprise zu zusätzlicher, durch die Form bedingter Emphase verhelfen (188f.). Diese und ähnliche Überlegungen fänden Anschluss an ältere Diskussionen zu Wagners Formsprache, vor allem zu dem, was in den zu Recht umstrittenen Publikationen von Alfred Lorenz »potenzierte Form « ${ }^{16}$ genannt wurde. Kritische und aktualisierende Auseinandersetzungen mit Lorenz haben zu verschiedenen
Zeiten mehrfach stattgefunden, ${ }^{17}$ und es wäre sicher eine Bereicherung, Vande Moorteles analytische Ansätze mit diesem Forschungsfeld der Wagner-Analyse zu verbinden. Man wundert sich fast ein bisschen, warum der Autor den Anschluss, eventuell auch in abgrenzender Absicht, an dieser Stelle vermieden hat.

\section{WIEDERHOLUNGSSTRATEGIEN UND REPRISENPROBLEME}

Die folgenden Kapitel »Open Ended Expositions« (191-221) und »Recomposed Recapitulations « (222-259) folgen trotz ihrer grundverschiedenen Inhalte einer gemeinsamen Logik: Beide zielen auf den Primat entwickelnder, auf das Finale ausgerichteter Form-Strategien, und zwar offenbar unter Vermeidung organizistischprozessualer Analysewerkzeuge. So behandelt Vande Moortele mit akribischer Genauigkeit sowohl statistisch in Tabellenform (210-213) als auch in Einzelanalysen die Standards des Wiederholens von Expositionen in Ouvertüren und Sinfonien. Während die Wiederholung in klassischen Sinfonien normal ist, findet sie in Ouvertüren fast nie statt. Das Ergebnis seiner Untersuchung ist die Einsicht in eine Abhängigkeit zwischen dem Vermeiden der Wiederholung zugunsten einer Verschleierung der Grenzen zwischen Exposition und Durchführung und der im Laufe des 19. Jahrhunderts steigenden Tendenz zu entwickelnden, schließlich auch Mehrsätzigkeit in die Einsätzigkeit integrierenden Werken wie Sinfonischen Dichtungen. Es scheint so, als habe die in Ouvertüren etablierte Norm des Nicht-Wiederholens auf die Kompositionspraxis von Sinfonien zurückgewirkt (213).

Unter »Wagners neuer Form» (222) versteht Vande Moortele eine Praxis der verkürzenden Reprise, die er in Wagners Essay De l'Ouverture bestätigt findet. Es handelt sich dabei grob gesagt um den direkten Anschluss der Coda an das Ende der Durchführung mit Überspringen von weiten Teilen der Reprise, wie Wagner es als Verbesserung der Form von Beethovens dritter Leonoren-Ouvertüre vorschlägt. ${ }^{18}$ Varianten dieser Strategie, und andere PhänoThorau 2003 und 2011, Jeßulat 2013.

18 Wagner 1914, 190. 
mene, die zunächst in Adornos MahlerMonografie als "Durchbruch ${ }^{19}$ bezeichnet wurden (224f.), und weitere Formen der veränderten, die bloße Wiederholung vermeidenden Ouvertüre werden sorgfältig diskutiert und in Detailanalysen von Wagners Komposition Eine Faust-Ouvertüre, Mendelssohns Ouvertüre zu Athalie und Donizettis Ouvertüre zu Don Pasquale veranschaulicht. Methodisch von Bedeutung ist, dass Vande Moortele zu diesem Zeitpunkt seiner Studie Leonard B. Meyers aus der Wahrnehmungspsychologie entlehnte Kriterien von "script" und "plan « heranzieht (226f.) und als Aktualisierung dessen verwendet, was Wagner in seiner Kritik mechanistischer Wiederholungen als "Wechsel « (für Mechanik) und »Entwicklung" (für die dramatische Idee) bezeichnet hat. ${ }^{20}$ Als "script « fungierten demnach die durch die Kompositionslehre und die Tradition gegebenen Strukturen sonatenhafter Formbildung, als "plan « die Erweiterungen, Abweichungen und scheinbar situativ bedingten Ausbrüche aus dieser Struktur. Da Finalprobleme, die starke Abweichungen vom "script" erfordern, wie das Vermeiden von Wiederholungen in der Reprise, in den früheren Prozess-Stadien Introduktion und Exposition in derart tiefgreifender Form noch nicht auftreten können, ist diese methodische Reflexion mit dem Rückgriff auf Meyer hier sinnvoll.

Als handele es sich bei der Abfolge der Kapitel des Buchs selbst um eine musikalische Formstrategie, endet die Studie mit der Betrachtung von »Apotheosen« (240-259), also Reprisenerweiterungen, in denen Thementransformationen aus Introduktion oder Exposition in überwältigender Grandezza am sozusagen letzten Schluss der Ouvertüre zurückkehren (240). Im Gegensatz zu Leonard B. Meyer, der die Gestalt von Apotheosen vor allem an sogenannten sekundären Parametern wie Dynamik, Tempo, Timbre und Klang festmacht, besteht Vande Moortele auf der Bedeutung der formalen Position ganz am Ende, »non plus ultra « (250). ${ }^{21}$

19 Adorno 1971, 152-154.

20 Vgl. Wagner 1914, 188-190.

21 In diesem Verständnis wäre also die C-DurVariante des Themas aus dem zweiten Satz der Fünften Sinfonie von Beethoven - so grandios

\section{»MUSICAL FORM FROM ROSSINI TO WAGNER«}

Das Vorhaben, die Werkzeuge der $>$ New Formenlehre $z u$ rekalibrieren und das analytische Corpus sowohl hinsichtlich der Gattung als auch des Zeitraums zu erweitern und zu differenzieren, ist in vieler Hinsicht gelungen. Durch die anti-kanonische Auswahl und Zusammenstellung paradigmatischer Analysen und den entschiedenen Verzicht auf gängige Analysekriterien nach dem Vorbild von Organismusmetaphern öffnet Vande Moortele neue Zugänge auf Ausprägungen romantischer Form und setzt dabei das Gemenge aus intern beschreibender Literatur aus dem Musikschrifttum des 19. Jahrhunderts und extern beschreibender Literatur aus der jüngeren amerikanisch geprägten Formenlehre neu zusammen. Die formale Eigenständigkeit reihender Formen wird in klugen und genauen Analysen bestätigt und auch die analytischen Annäherungen an Werke, die einem organizistischen Ideal entsprächen, werden aus der nüchternen, eher simplifizierenden Perspektive dieser modifizierten New Formenlehres auf angenehme Weise sentkrustet،.

Kritisch anzumerken ist höchstens der Umgang mit bestimmten Diskursen der möglicherweise noch nicht ganz verjährten musikwissenschaftlichen Formenlehre, die für den Forschungsgegenstand dieser Studie durchaus greifen würden, aber zum Teil nicht einmal erwähnt werden. So ist es verständlich, dass der Autor sein Corpus gegenüber den Sinfonischen Dichtungen und ihrer musikwissenschaftlichen Rezeption abgrenzt. Angesichts der Sorgfalt, mit der er die Differenzierung zu den früheren Formen der Sinfonia, zu ersten Sätzen von Instrumentalkonzerten und zu klassischen Sinfonien vollzieht, ist es dennoch verwunderlich, dass auf die inhaltlich gewichtigen Arbeiten von Dahlhaus und Norbert Mil$\operatorname{ler}^{22} \mathrm{zu}$ den Formproblemen der Sinfonischen Dichtung als Weiterentwicklung der Konzert-

und erhaben sie auch in Besetzung und Modulation ist - keine Apotheose.

22 Besonders Miller 1975; Dahlhaus/Miller 2007. 
ouvertüre so gut wie gar nicht eingegangen wird. ${ }^{23}$

Der zweite Kritikpunkt schließt direkt daran an: Das Etikett sorganizistisch Spektrum existierender Forschungsfelder, das in seiner Breite sowohl die künstlerischen und wissenschaftlichen Werke E. T. A. Hoffmanns, die zum Teil spinozistische Idee einer natura naturans als auch Goethes >Metamorphose der Pflanzen `Neuen Mythologie، schließlich auch den Übergang in eine Ästhetik des Industriezeitalters einschließt. Eine derart weite und zudem tief in der idealistischen Philosophie verankerte Plattform wie die Idee des Organischen in der Ästhetik des 19. Jahrhunderts durch das sehr schmale Nadelöhr nur weniger musiktheoretischer Schriften, vor allem von Marx, Johann Christian Lobe und Gottfried Weber, zu drängen, schließt auch jüngere Diskurse wie die Auseinandersetzung mit Formproblemen bei Beethoven aus. So sind z. B. Strategien wie das Überblenden von Introduktion und Exposition schon bei Dahlhaus in der Analyse von Beethovens Klaviersonate op. 31/2 beschrieben, der wiederum auf die Entstehung dieses Gedankens bei August Halm verweist. ${ }^{24}$ Es ist verständlich, wenn ein höchst origineller und eigenständiger Ansatz verunklarende Berührungen mit den Diskursen des Beethoven-Paradigmas vermeiden möchte, allerdings hätte eine wenigstens exemplarisch erfolgte Vernetzung zwischen der überaus dichten deutschsprachigen Formenlehre-Diskussion der 1970er und 80er Jahre mit dieser Differenzierung der `New Formenlehre` zum Gewinn der Studie sicher beigetragen. Dass z. B. „Sonatendenken « ${ }^{25}$, „Zeiten der Form ${ }^{26}$, spoetische Idee ${ }^{27}$ etwas anderes bedeuten als »localized sonaticization«, »begin-

23 Auch in Vande Moorteles älteren Schriften zu Formproblemen der Sinfonischen Dichtung wird die einschlägige deutschsprachige $\mathrm{Mu}$ sikwissenschaft zugunsten einer ausführlichen Diskussion von Hepokoski nur knapp rezipiert. Eine Auseinandersetzung mit Dahlhaus findet punktuell statt, mit Danuser oder Miller eigentlich nicht. Vgl. Vande Moortele 2008, 2009.

24 Vgl. Dahlhaus 1980b, 311. ning-middle-end» (112) oder "plan«, da sie von grundlegend anderen analytischen und kulturgeschichtlichen Voraussetzungen ausgehen, aber ähnliche Momente formaler Verfügbarkeit und Orientierung berühren, würde die neuen Beschreibungsmodelle aufwerten und den zeiträumlichen Vorstellungen der >New Formenlehre` zu etwas mehr Anschlussfähigkeit an die oft weniger geradlinigen und von reflexiven Bewegungen durchzogenen Vorstellungen idealistisch-romantischer Theorie verhelfen.

Da die Applikation neuer Analyse-Modelle immer einen Eingriff in die bewusste Wahrnehmung von Musik bedeutet, kann nicht genug hervorgehoben werden, dass Vande Moortele sozusagen implizit mit einer höranalytischen Ausgangssituation arbeitet, was nicht selbstverständlich ist. Für die einzelnen analytischen Schritte und die Orientierung innerhalb der musikalischen Form spielt ein Zugang, der nicht nur am Notentext, sondern mit Höreindrücken und erinnernder Re-Organisation, mit der Wirkung von Zeit-Dauern und Beschleunigung von Form arbeitet, eine überaus relevante Rolle. Für diese Studie sehr wichtige argumentative Zwischenergebnisse wie die Multivalenz von Formteilen an den Übergängen wären in einer sreinen` Textanalyse möglicherweise nicht existent.

Insgesamt vereint das Buch in beeindruckender formaler Dramaturgie eine Fülle an Material und kluge, ökonomisch präsentierte Analysen unter der Hüllkurve eines sinnfälligen und notwendigen Weiterdenkens aktueller Formenlehre und leistet damit einen wichtigen Beitrag zur Aufarbeitung und Aktualisierung der Musikgeschichte des 19. Jahrhunderts.

Ariane Jeßulat
25 Danuser 2003, 312.

26 Halm 1916, 48.

27 Vgl. Schindler 1860, 195; Bekker 1911, 2017; Dahlhaus 1966; 21; Renger 2003. 


\section{Literatur}

Adorno, Theodor W. (1971), Mahler. Eine musikalische Physiognomik [1960], in: ders., Die musikalischen Monographien, Frankfurt a. M.: Suhrkamp, 149-319.

Agawu, Kofi V. (1991), Playing with Signs: A Semiotic Interpretation of Classic Music, Princeton: Princeton University Press.

Bekker, Paul (1911), Beethoven, Berlin und Leipzig: Schuster \& Loeffler.

Bonds, Marc Evan (1991), Wordless Rhetoric: Musical Form and the Metaphor of Oration, Cambridge (MA): Harvard University Press.

Boulez, Pierre (1963), Musikdenken heute, Mainz: Schott.

Brinkmann, Reinhold (1972), „Drei Fragen stell' ich mir frei: Zur Wanderer-Szene im 1. Akt von Wagners Siegfried", Jahrbuch des Staatlichen Instituts für Musikforschung 5, 120-162.

Caplin, William E. (1998), Classical Form: A Theory of Formal Functions for the Instrumental Music of Haydn, Mozart, and Beethoven, New York: Oxford University Press.

Dahlhaus, Carl (1965), „Wagners Begriff der >dichterisch-musikalischen Periode««, in: Beiträge zur Geschichte der Musikanschauung im 19. Jahrhundert, hg. von Walter Salmen, Regensburg: Bosse, 179-187.

Dahlhaus, Carl (1966), »Musica Poetica und musikalische Poesie«, Archiv für Musikwissenschaft 23/2, 110-124.

Dahlhaus, Carl (1969), „Formprinzipien in Wagners Ring des Nibelungen«, in: Beiträge zur Geschichte der Oper, hg. von Heinz Becker, Regensburg: Bosse, 95-129.

Dahlhaus, Carl (1980a), „Cantabile und thematischer Prozess. Der Übergang zum Spätwerk in Beethovens Klaviersonaten«, Archiv für Musikwissenschaft 37/2, 81-98.

Dahlhaus, Carl (1980b), »Zur Formidee von Beethovens d-Moll-Sonate Op. 31, 2 «, Die Musikforschung 33/3, 310-312.

Dahlhaus, Carl (1988), Klassische und romantische Musikästhetik, Laaber: Laaber.

Dahlhaus, Carl / Norbert Miller (2007), Europäische Romantik in der Musik. Oper und sin- fonischer Stil 1800-1850. Von E. T. A. Hoffmann zu Richard Wagner, Stuttgart: Metzler.

Danuser, Hermann (2003), »Form - Formation Transformation«, in: ders., Gesammelte Vorträge und Aufsätze, Bd. 1, hg. von Hans-Joachim Hinrichsen, Christian Schaper und Laure Spaltenstein, Schliengen: Argus, 306-326.

Gossett, Philipp (1979), »The Overtures of Rossini «, 19th-Century Music 3/1, 4-13.

Halm, August (1916), »Musikdrama und Sonatenform«, in: ders., Von Grenzen und Ländern der Musik, München: Georg Müller, 47-57.

Heinz, Marion (2002), »Idealisierung des Weiblichen: Wilhelm von Humboldt«, in: Philosophische Geschlechtertheorien, hg. von Sabine Doyé, Marion Heinz und Friederike Kuster, Stuttgart: Reclam, 276-282.

Hepokoski, James / Warren Darcy (2006), Elements of Sonata Theory: Norms, Types, and Deformations in the Late-Eighteenth-Century Sonata, New York: Oxford University Press.

Humboldt, Wilhelm von (1795), „Über den Geschlechtsunterschied und dessen Einfluß auf die organische Natur «, Die Horen 1/2, 99-132.

Jeßulat, Ariane (2013), Erinnerte Musik. Der Ring des Nibelungen als musikalisches Gedächtnistheater, Würzburg: Königshausen \& Neumann.

Jeßulat, Ariane (2015), „Synchron und diachron. Zum Zusammenhang zwischen Kontrapunkt und Prozessualität in romantischer Formensprache«, ZGMTH 12/1, 99-128. https://doi.org/10.31751/803 (15.12.2019)

Kawohl, Friedemann (2005), „Organismusmetaphern«, in: Musiktheorie, hg. von Helga de la Motte-Haber und Oliver SchwabFelisch, Laaber: Laaber, 156-167.

Krummacher, Friedhelm (1980), „Synthesis des Disparaten - Zu Beethovens späten Quartetten und ihrer frühen Rezeption«, Archiv für Musikwissenschaft 37/2, 99-134.

Lorenz, Alfred (1924), Der musikalische Aufbau des Bühnenweihfestspiels Der Ring des Nibelungen, Berlin: Hesse, Reprint Tutzing: Schneider. 
Marx, Adolph Bernhard (1845), Die Lehre von der musikalischen Komposition, Bd. 3, Leipzig: Breitkopf \& Härtel.

Miller, Nobert (1975), »Musik als Sprache. Zur Vorgeschichte von Franz Liszts symphonischen Dichtungen«, in: Beiträge zur musikalischen Hermeneutik, hg. von Carl Dahlhaus, Regensburg: Bosse, 223-287.

Rellstab, Friedrich (1834), [Recension der »Hebriden-Ouvertüre«], Iris im Gebiete der Tonkunst 5/13, 49-50.

Renger, Jens (2003), Im Labyrinth der "poetischen Idee». Werk- und rezeptionsästhetische Studien zur Instrumentalmusik des frühen Beethoven, Sinzig: Studio.

Rigaudière, Marc (2009), La théorie musicale germanique du XIXe siècle et l'idée de cohérence, Paris: Société française de musicologie.

Schindler, Anton (1860), Biographie von Ludwig van Beethoven, 3. Auflage, Münster: Aschendorff.

Schmalfeldt, Janet (2011), In the Process of Becoming: Analytic and Philosophical Perspectives on Form in Early NineteenthCentury Music, New York: Oxford University Press.

Thorau, Christian (2003), »Vielfalt, Polyphonie, Implikation und Prozeß. Formgestaltung im Musikdrama Richard Wagners«, in: Der
"Komponist" Richard Wagner im Blick der aktuellen Musikwissenschaft. Symposion Würzburg 2000, hg. von Ulrich Konrad, Wiesbaden: Breitkopf \& Härtel, 141-150.

Thorau, Christian (2011), »Formgestaltung und szenische Verkörperung in der WandererMime-Szene des ersten Siegfried-Aufzugs. Zur Relation von Musikanalyse und Inszenierungsgeschichte«, in: Wagners Siegfried und die (post-)heroische Moderne. Beiträge des Hamburger Symposiums 22.-25. Oktober 2009, hg. von Tobias Janz, Würzburg: Königshausen \& Neumann, 217-234.

Vande Moortele, Steven (2008), „Beyond Sonata Deformation: Liszt's Sinfonic Poem Tasso and the Concept of Two-Dimensional Sonata-Form«, Current Musicology 86, 41-62.

Vande Moortele, Steven (2009), TwoDimensional Sonata Form: Form and Cycle in Single-Movement Instrumental Works by Liszt, Strauss, Schoenberg, and Zemlinsky, Leuven: Leuven University Press.

Wagner, Richard (1914), „Über Franz Liszts symphonische Dichtungen«, in: ders., Sämtliche Schriften und Dichtungen, Bd. 5, hg. von Wolfgang Golther, Berlin: Hesse, 188195.

Jeßulat, Ariane (2019): Steven Vande Moortele, The Romantic Overture and Musical Form from Rossini to Wagner, Cambridge: Cambridge University Press 2017. ZGMTH 16/2, 199-208. https://doi.org/10.31751/1026

(C) 2019 Ariane Jeßulat (ajessulat@aol.com) Universität der Künste Berlin

Dieser Text erscheint im Open Access und ist lizenziert unter einer Creative Commons Namensnennung 4.0 International Lizenz.

This is an open access article licensed under a

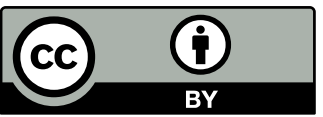

Creative Commons Attribution 4.0 International License.

eingereicht / submitted: 17/11/2019

angenommen / accepted: 19/11/2019

veröffentlicht / first published: 23/12/2019

zuletzt geändert / last updated: 23/12/2019 